\title{
Phase-Referencing Observations of Galactic Circumstellar Masers
}

Huib Jan van Langevelde

Joint Inst. for VLBI Europe, Postbus 2, 7990 AA Dwingeloo, the Netherlands

Loránt O. Sjouwerman

Onsala Rymdobservatorium, S-43992, Sweden

Philip J. Diamond

National Radio Astronomy Observatory, Socorro, NM 87801, U.S.A.

Richard T. Schilizzi

Joint Inst. for VLBI Europe, Postbus 2, 7990 AA Dwingeloo, the Netherlands

Alain Baudry

Observatoire de Bordeaux, BP 89, F-33270 Floirac, France

Abstract. Two projects are discussed in which a phase referencing technique is used to determine stellar parallaxes and/or proper motions by means of the circumstellar masers. The observations are special in the sense that both continuum and line sources need to be detected. To obtain stellar motions assumptions on the location of the maser with respect to the star need to be made.

\section{Parallax of the Nearby Mira Variable U Her}

We have observed the circumstellar $1667 \mathrm{OH}$ maser in U Her with the VLBA. This object is chosen as it is one of the closest Mira variables with known $\mathrm{OH}$ maser emission. We selected the $\mathrm{OH}$ transition, although at a low frequency, because observations suggest that the brightest blue--shifted emission is the stellar image amplified by the circumstellar maser (Sivagnanam et al. 1990).

The observations employ a phase referencing technique, using two extragalactic calibrators from a catalogue by Patnaik et al. (1992). These sources are $\lesssim 3^{\circ}$ away from the target and we use $5-7$ min cycles. The VLBA allows us to observe this with a mixed bandwidth mode. Both polarizations were recorded on the line using oversampled $500 \mathrm{kHz}$ bands; several $4 \mathrm{MHz}$ bands were recorded simultaneously at adjacent frequencies to obtain sufficient continuum sensitivity. We find that the phase connection during all three epochs, spanning the July 1994 to August 1995 period, works very well, possibly because of the solar minimum. Without special processing we can image the maser with the phase, delay and rate solutions obtained on the continuum channels. From measuring the distance between the two calibrators at the three epochs of the observations we estimate systematic effects to be $\lesssim 0.7$ mas.

It is interesting to compare the three radio observations with the Hipparcos results on this object. Comparison of the absolute maser radio position and the optical star is limited by the positional accuracy of the radio position of the extragalactic calibrators to \pm 15 mas. A proper motion is measured from the VLBA data $\mu=-0.0247,-0.0122 \mathrm{mas} / \mathrm{yr}$ in $\mathrm{RA}$ and dec., respectively. This is roughly consistent with the Hipparcos results. With 3 epochs only it is hard to determine a parallax from the VLBI data. The Hipparcos data are not very 
conclusive on the distance to this object, because it is relatively faint. Using an ad-hoc estimate for the U Her distance of $350 \mathrm{pc}$ and the fitted radio proper motion, positional residuals of 1-2 mas remain. This seems to indicate a problem with our basic assumption that the maser motion reflects the stellar motion. Possibly gas motions in the shell are important, or an additional component to the motion is needed. The maser structure also shows indications that U Her is not a single star, but may have more components inside a common envelope.

\section{Stellar Proper Motions in the Galactic Center}

A second project attempts to detect stellar proper motions in the Galactic center. This is of importance, not only to study the possible presence of a massive black hole, but also to study the potential of the Galactic bulge and the dynamic history of the Galaxy. Already stars with large radial velocities are known: a velocity of $400 \mathrm{~km} / \mathrm{s}$ corresponds to $10 \mathrm{mas} / \mathrm{yr}$. At the same time a kinematic study with proper motions will yield an independent estimate of the distance to the Galactic center. From previous surveys (e.g., Lindqvist et al. 1992, Sjouwerman et al. 1997) a large number of AGB stars are known through their circumstellar $\mathrm{OH}$ masers. Because of the large interstellar scattering toward the Galactic center (e.g., van Langevelde et al. 1992), we are forced to use $\mathrm{H}_{2} \mathrm{O}$ and especially $\mathrm{SiO}$ masers. A targeted survey for this was carried out with the VLA (Sjouwerman et al., in preparation).

We have carried out observations in which we use $\operatorname{Sgr~A}^{\star}$ as a phase calibrator and cycle every 40 s to $\mathrm{SiO}$ masers within $\lesssim 15^{\prime}$. With just the inner 6 VLBA antennas we failed to make any detections. We conclude that the inclusion of the VLA is crucial for this experiment. First, the use of the VLA allows us to check whether the variable masers are bright enough during the observations. Moreover, the VLA adds the necessary sensitivity, even though its use is quite complicated for fast phase referencing. In order to accommodate the $20 \mathrm{~s}$ source switching we divided the 13 VLA antennas with $\mathrm{Q}$-band receivers into two groups. One group, connecting to one IF input on the VLBA recorder continuously observes the maser target, the other, writing a different "polarization" on $\operatorname{Sgr} \mathrm{A}^{\star}$, the reference source.

On two subsequent epochs we had detections for two different stars out of a handful of attempts. It shows that 1 mas accurate positions of the masers in the Galactic center is possible. However, to obtain a significant sample of proper motions requires elaborate monitoring.

Acknowledgments. HJvL acknowledges support for his research by the European Union under contract CHGECT920011. The National Radio Astronomy Observatory is a facility of the NSF, operated under a cooperative agreement by Associated Universities, Inc.

\section{References}

Lindqvist, M., et al., 1992. $A \mathscr{E} A S, \mathbf{9 2}, 43-62$.

Patnaik, A. R., et al., 1992. MNRAS, 254, 655-676.

Sivagnanam P., et al., 1990. $A \& A, 229,171-176$.

Sjouwerman, L. O., et al., 1997. A\&AS, in press.

van Langevelde, H. J., et al., 1992. $A p J, \mathbf{3 9 6}, 686-695$. 